\title{
ILCEA
}

Revue de l'Institut des langues et cultures

d'Europe, Amérique, Afrique, Asie et Australie

$21 \mid 2015$

Discours politique et culturel dans la Russie

contemporaine

\section{CESC et analyse du discours soviétique}

The CESC and the Analysis of Soviet Discourse

\section{Alexandre Bourmeyster}

\section{OpenEdition}

Journals

Édition électronique

URL : http://journals.openedition.org/ilcea/3017

DOI : 10.4000/ilcea.3017

ISSN : 2101-0609

\section{Éditeur}

UGA Éditions/Université Grenoble Alpes

Édition imprimée

ISBN : 978-2-84310-294-3

ISSN : 1639-6073

\section{Référence électronique}

Alexandre Bourmeyster, «CESC et analyse du discours soviétique », ILCEA [En ligne], 21 | 2015, mis en ligne le 01 février 2015, consulté le 30 avril 2019. URL : http://journals.openedition.org/ilcea/3017 ; DOI : 10.4000/ilcea.3017

Ce document a été généré automatiquement le 30 avril 2019

(C) ILCEA 


\section{CESC et analyse du discours soviétique}

The CESC and the Analysis of Soviet Discourse

Alexandre Bourmeyster

1 Mes premières relations avec le « discours soviétique » datent des années 1964-1974, de l'époque où je coopérais avec le Centre de recherches sur l'URSS et les pays de l'Est, créé par le professeur Michel Mouskhély (1903-1964) à la Faculté de droit et de sciences politiques et économiques de Strasbourg. Il publiait, avec l'appui du CNRS, un Annuaire de l'URSS et des mensuels, des recueils d'abstracts des principales publications soviétiques. Mon secteur d'activité couvrait Voprosy filosofii et Kommunist. J'éprouvais une étonnante facilité pour rédiger mes abstracts à partir de textes verbeux, encombrés de citations et d'effets oratoires; la rédaction des articles de synthèse pour l'Annuaire exigeait, en revanche, de l'attention et de la prudence, afin d'appréhender des données suggérées implicitement ou présupposées. Faute d'outil d'analyse approprié pour les déchiffrer, je me gardais néanmoins de chercher à "lire entre les lignes », une prospection pourtant couramment pratiquée par des soviétologues qui se prétendaient avisés.

2 À cette époque, enseignant de littérature comparée à Lyon, je participais à des séminaires où s'imposait le structuralisme inspiré par le Cours de linguistique générale de Ferdinand de Saussure, la différence entre langue et parole, la distinction entre signifiant et signifié, l'opposition entre synchronie et diachronie; cette perspective de recherche était passionnante, mais je n'y trouvais guère d'application pour analyser la phraséologie soviétique. En outre, j'achevais alors ma thèse de doctorat d'État sur Stankevič, promoteur de l'hégélianisme en Russie, une pensée dialectique aux antipodes de la linguistique des structuralistes. Les choses ont évolué lorsque je me suis retrouvé, en 1973, à Grenoble, à la tête du Département des langues slaves, au temps des grandes réformes post-68, notamment l'introduction de la filière pré-professionnelle MLEA. Elle ne conservait de l'enseignement des langues vivantes que la traduction, la compréhension-expression et la civilisation, nourrie, non plus par la littérature, mais par la presse quotidienne du pays concerné. En russe, la Pravda et les Izvestia devaient nous rendre les mêmes services que le Spiegel, le NY Times, la Stampa, aux collègues 
germanistes, anglicistes, italianistes. Hélas, nous nous sommes vite heurtés, à la fois, à l'aversion de nos étudiants et à notre propre incapacité de traiter de façon vivante des problèmes déjà résolus sans appel, de façon idéologique, dans les articles consultés. Il nous fallut revenir précipitamment à des textes littéraires, à des auteurs contemporains, Paoustovski Trifonov, Solooukhine, Kazakov, pour retrouver un terrain fertile, propre aux débats en civilisation.

Restait à faire un constat: la presse soviétique était inutilisable pédagogiquement au premier degré, celui de la communication. Que fallait-il en faire ? L'ignorer, l'éliminer de notre cursus, à l'exemple de nos collègues russisants dans d'autres universités ? Ou l'aborder au second degré, d'un point de vue critique, universitaire, sans a priori politique? Mais avec quels outils? Après avoir goûté à l'ouverture d'esprit dans la recherche à Lyon, je me sentais un peu isolé et délaissé à Grenoble. Mes collègues du Département se spécialisaient dans la littérature soviétique ou dans la linguistique, en dehors de cette problématique; nous appréciions néanmoins ensemble des œuvres majeures comme Morphologie du conte de Vladimir Propp ou Problèmes de la poétique de Dostoievski de Mikhaïl Bakhtin. De mon côté, je découvrais A. J. Greimas, sa sémiotique, son programme narratif, son schéma de communication hérité de Jakobson et ses actants, énonciateurs, narrateurs et interlocuteurs. Je suivais ses séminaires à l'École pratique des hautes études à Paris et j'appliquais son carré sémantique au dilemme vécu par Raskolnikov (voir annexe, ci-dessous). Greimas remarqua à ce propos que j'avais l'esprit dialectique, ce qui me conforta dans l'idée qu'il était lui-même un dialecticien investi dans un projet sémiotique. Il me recommanda de suivre au Centre théologique de Meylan les journées d'études consacrées à l'analyse sémiologique de textes de l'Ancien et du Nouveau Testaments. Je les ai suivies régulièrement en compagnie de dames catholiques ou protestantes, soucieuses de renouveler l'approche de ces écrits archi-connus, dans leurs activités de catéchèse, auprès de leurs églises respectives. Cet exercice tonique, passionnant me suggéra l'idée de l'appliquer à l'analyse de la presse soviétique. Approuvant ce projet, Claude Robert, directeur du Centre d'études slaves contemporaines (CESC), m'en céda bientôt la direction. Il l'avait consacré jusqu'alors à des travaux en grammaire et en linguistique. En 1978 débuta le séminaire de $3^{\mathrm{e}}$ cycle sur le «Discours soviétique » avec des séances hebdomadaires, le jeudi après-midi.

Dès le début, j'ai trouvé un allié de poids en la personne de Patrick Sériot, professeur de russe dans un lycée de la banlieue de Grenoble. Il s'était fait remarquer par une étude pertinente sur le militarisme de nombreuses citations dans le renommé dictionnaire d'Ojegov. Il convenait de définir l'objet de nos recherches, dans le cadre d'un séminaire consacré désormais à l'analyse de la presse soviétique. Langue ou parole ? Langue de bois, newspeak ou procès, message? A priori, nous avons repoussé les approches «non scientifiques ", celles qui consistaient à lire entre les lignes, à démasquer le faux, le «mensonger» au profit du «vrai »; il fallait lire les lignes, considérer l'écrit étudié comme un discours assumé par un sujet parlant, et accessible, en tant qu'énoncé et en tant qu'énonciation, à l'analyse linguistique et sémiotique. Avec le recul du temps, je dois reconnaître qu'en dépit de convictions communes, nos références étaient différentes. Je m'investissais entièrement dans les grilles de lecture à la Greimas pour identifier dans les discours officiels, publiés dans la Pravda, le destinateur et le destinataire, le sujet opérateur, l'objet de sa performance, son adjuvant et son obstacle, l'anti-sujet, afin de reconstituer une performance, au final, glorifiée ou blâmée. Inspiré par V. Propp, par C. Levi-Strauss, le programme narratif de Greimas, enrichi et confirmé par tant de contes, 
de mythes et de légendes, pouvait être actualisé de nos jours, par les BD, notamment par les Aventures d'Astérix le Gaulois.

5 Les perspectives de Patrick Sériot étaient différentes, il se référait à Michel Pêcheux (Les Vérités de La Palice) qui fondait sa réflexion sur la notion de coupure épistémologique, héritée de Bachelard. Dans ses cours, Althusser la popularisa par son étude des « conditions de production ", du Capital de Marx : afin d'accéder au statut de science de l'histoire et de la société, le marxisme devait s'émanciper, dans une lutte permanente, de l'idéalisme. Pêcheux en déduisait que l'instrument de la lutte contre l'idéologie, l'analyse du discours, était la partie constitutive de tout projet scientifique. Il ne suffisait pas de remplacer un discours (idéologique) par un autre, il fallait opérer une coupure épistémologique. Obstacles à la constitution d'une science, les idéologies avaient, selon Pêcheux, une forme empirique, avec une fonction technique, sémantique, la coïncidence du signifiant avec le signifié, et une forme spéculative, politique, avec l'assemblage des significations entre elles, sous la forme du discours. Dans leur état actuel, les «sciences sociales » étaient inaptes à déceler la forme spéculative des idéologies, car elles n'abordaient le discours que sous une forme empirique. La linguistique de Saussure s'était constituée en tant que science par une coupure épistémologique, en opposant langue et parole, mais elle avait " négligé » de donner du sens à la parole, au discours, laissant place libre aux idéologies. À qui confier la lecture des textes, pour leur donner un sens? À un sujet? Selon Pêcheux, ce « lecteur » était tout juste capable de s'identifier à l'un des points de vue idéologiques exprimés, explicitement ou implicitement, s'il ignorait les "conditions de production » du discours qu'il analysait, un interdiscours. Là, dans l'interdiscours, s'affrontaient discours dominant, contre-discours, traces de discours d'un Autre. Il n'était pas question de recourir à une lecture plurielle, à la polyphonie, à la poétique de Dostoïevski, à la manière de Bakhtine, mais de réaliser une coupure épistémologique, la désubjectivisation d'un sujet, à la fois possesseur du sens qu'il déchiffrait et dépossédé de ce sens au profit d'une interprétation qui suivait à la trace le procès de l'interdiscours lui-même.

6 Cette vision théorique de l'analyse du discours permettait surtout de révéler les obstacles méthodologiques qu'il fallait surmonter pour éviter les approches «subjectives» de la phraséologie soviétique, comme celle de Michel Heller qui opposait le vrai au faux, la langue russe à la langue soviétique, une Novlangue, langue officielle d'un tiers de l'humanité, ou celle d'Alain Besançon traitant de logocratie une langue de bois capable de créer une sur-réalité d'existence exclusivement verbale, le règne du mensonge pur, ou encore celle de Françoise Thom qui prétendait traiter grammaticalement la "langue de bois ", une expression journalistique, polémique, sans fondement linguistique. Dans le cadre du schéma de communication, cette notion d'interdiscours me permettait de concevoir un discours soviétique en tant que champ de bataille où, en dehors des propos de l'énonciateur officiel, sont décelables, sous forme de shifters, selon la terminologie de Jakobson, les traces d'une voix anonyme, celle d'un autre énonciateur. Patrick Sériot, lui, percevait autre chose, les signes d'une manipulation dans le discours officiel, destinée à effacer les traces de toute tentative antérieure de contestation.

Retenons les étapes marquantes dans les activités du CESC :

1981: parution du premier numéro des Essais sur le discours soviétique (sémiologie, linguistique, analyse discursive). Il débutait par une préface affirmant que l'analyste doit s'intéresser à la façon dont se construit le discours, plutôt qu'à sa signification supposée, au comment plutôt qu'au pourquoi et au quoi ; il contenait des articles mettant à l'épreuve une pléthore de procédés d'analyse. Chez Patrick Sériot: dé-nominalisation, 
transformations de l'actif au passif et inversement, transformations des énoncés en classes d'équivalence, à propos d'un discours sur la science de Brejnev. Chez moi, traduction des divers énoncés de la nouvelle Constitution soviétique en formules canoniques, propres à l'analyse sémiologique de Greimas, pour dégager les actants, les modalités d'une manipulation destinée à transformer la compétence du sujet (le citoyen soviétique) en performance.

1981: participation du CESC au Colloque international de linguistique slave à Aix-enProvence sous la présidence de Paul Garde, une reconnaissance par nos pairs du caractère scientifique de nos recherches.

1982: parution du $\mathrm{n}^{\circ} 2$ des Essais avec une participation élargie. Aux six auteurs du premier numéro, P. Sériot, A. Bourmeyster, G. Leyzieux, R. Roche, A. Berelovitch, R. Best, s'ajoutaient les noms de J. Breuillard, Ch. Bourg, Armelle Groppo. Dans ce numéro, à propos d'une condamnation de la ligne politique du parti communiste italien dans la Pravda, j'appliquais le schéma de communication de Jakobson, revu par Greimas, pour faire entendre la voix de l'Autre condamné pour révisionnisme et trahison, au nom de la fidélité aux thèses officielles, ce que j'appelais le skaz.

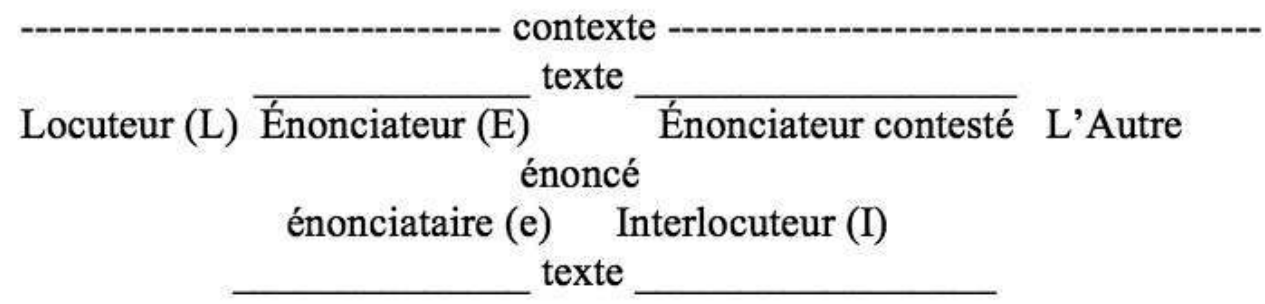

Robert Best notait que le discours-réplique (le discours anti-anti-soviétique) réservait à l'Autre un crédit suffisant pour se discréditer lui-même. De son côté, tout en présentant la meilleure illustration de la langue de bois, le "code universel du discours», une facétie d'étudiants, parue de façon insolite dans la Gazette de Varsovie en 1981, P. Sériot soulignait l'abondance des nominalisations et mettait en garde contre la confusion entre langue et discours, des considérations qu'il allait bientôt développer dans sa thèse de doctorat.

1983 : le 4 juin, soutenance à Grenoble de la thèse de doctorat de P. Sériot: Préliminaires linguistiques à une analyse du discours politique soviétique : les relations prédicatives non verbales . Au jury: président, Jacques Veyrenc, devenu un collaborateur fidèle des Essais, J. Breuillard, M. Pêcheux et moi, le directeur de thèse. Ma direction avait consisté surtout à proposer un sujet de recherche. L'introduction de l'ordinateur à l'Université rendait possible son application en sciences humaines et sociales. Premier usage, quantitatif, le comptage ; on évaluait la richesse de la langue de Corneille et son appauvrissement avec l'âge, on comparait l'usage du terme liberté par Giscard d'Estaing, avec celui de peuple, par Mitterrand. Je proposais à P. Sériot de comparer le discours de Khrouchtchev à celui de son successeur, Brejnev. Il s'attela, pendant plus d'un an, à cette tâche austère auprès, non pas d'un portable, alors inexistant, mais du "dinosaure » installé sur le campus à l'usage des "sciences dures"; des créneaux horaires rares, souvent incommodes, des résultats décevants et un résultat mitigé. Sériot, désolé, ne constatait aucun changement notable, pas de coupure épistémologique entre les discours de Khrouchtchev et de Brejnev " écrits tous les deux par la même main ", mais il retenait la surabondance des génitifs, des nominalisations, la preuve avérée, grâce à l'informatique, d'une manipulation opérée par un sujet anonyme, destinée à effacer toute référence à des 
prédications antérieures. Cette enquête lexicale, syntaxique, sur les conditions de production du discours soviétique aboutissait, sans définition a priori, à différencier langue et discours et mettait fin à une vieille polémique entre collègues.

Elle ne mettait pas fin, hélas, malgré tous mes efforts, à une animosité qui ferma à P. Sériot l'entrée qu'il méritait amplement dans notre Département à Grenoble. Installé à Lausanne, où il se distingua vite par ses travaux, il n'en poursuivit pas moins, pendant des années, sa coopération avec le CESC. Il axait ses réflexions sur la « Grande langue russe »: était-elle objet du croire ou du savoir ? Pourquoi était-elle " grande » ? Parce qu'elle était une pièce fondamentale du système soviétique? Parce qu'elle était une langue universelle ? Trop parfaite, ne risquait-elle pas de devenir une non-langue?

14 À la soutenance, Michel Pêcheux avait manifesté un grand intérêt pour cette " première " et avait prédit à P. Sériot un grand avenir. Nous ne pûmes, hélas, bénéficier de sa compétence car, peu après, il mettait fin à ses jours. Triste récapitulatif : en 1985, le 13 août, nous perdions, dans une mort subite, notre aîné, Jacques Veyrenc. Ce linguiste éminent manifestait un appui chaleureux à notre séminaire, nous attendions beaucoup de lui, notamment après la publication dans les Essais de son étude, "La néo-langue de George Orwell comme auto-parodie ». En 1986, mettait fin à ses jours notre compagnon et ami, Robert Best, un esprit rigoureux et compétent, autant philosophe que sociologue, autant linguiste que sémiologue, toujours apte à s'emparer de sujets originaux, à les traiter de façon saisissante. Une perte irréparable.

Le CESC continue néanmoins d'exister, il gagne de nouveaux collaborateurs. En 1985, pour pouvoir participer au $3^{\mathrm{e}}$ Congrès mondial d'études soviétiques et est-européennes, à Washington (30 octobre-3 novembre 1985), je monte un panel international avec un Français, R. Bautier, une Polonaise, B. Rogulska, un Américain, M.-E. Urban. Ce congrès est un rassemblement gigantesque où, pour capter un public, il est indispensable de s'exprimer dans un anglais correct. Que d'efforts à faire! J'expose à cette occasion ma théorie du skaz, un terme emprunté à la linguistique russe qui définit un récit mené, dans son style propre, par un narrateur, que l'auteur ou le locuteur intègre dans son énoncé personnel, sans user de guillemets ou d'autre signe distinctif. Leskov fut le maître de cette expression littéraire au XIX ${ }^{e}$ siècle. Mon skaz, c'est un récit légendaire, une interprétation officielle de la naissance et du destin de l'URSS, il s'inscrit implicitement sans guillemets, dans tout énoncé, article, discours ou œuvre littéraire soviétique. Tout citoyen soviétique est éduqué pour dénoncer et corriger toute éventuelle dérive, surtout dans les propos des étrangers. Je fais l'expérience de cette pratique avec les étudiants soviétiques que nous recevons chaque année en stage de plusieurs mois à l'université. Élèves des Instituts de traduction et d'interprétation, ils suivent avec moi un cours de traduction des discours les plus officiels qui soient, ceux de Brejnev, de Souslov ou de Gromyko, précisément le corpus de nos séances d'analyses sémiologiques au séminaire du CESC! Nous ressentons ensemble, mes étudiants et moi, la difficulté qu'il y a à transposer ces insupportables propos dans la belle langue française. Je tente parfois, à titre de test, d'assouplir cette prose au risque d'entacher le skaz. Aussitôt dans l'assistance, un étudiant se doit d'intervenir pour me rectifier. Mais nous ne sommes pas dupes ni eux, ni moi ; nous sommes complices dans un exercice formel qui relève du rituel. Ces étudiants soviétiques constituent une élite appelée, dans leur avenir, à exploiter leurs acquis dans des pays francophones, souvent contre les intérêts de la France, mais susceptibles aussi d'être gagnés par un esprit critique envers leur idéologie; ils se doutent de l'existence de notre séminaire, mais je me garde bien de les informer, de peur de les compromettre. En fait, ce 
skaz est destiné à légitimer un PCUS et un État soviétique ébranlés par la déstalinisation, il répond au besoin d'un consensus, à l'entretien d'une illusion, l'unanimisme au sein de l'opinion, l'harmonie entre le pouvoir et la population. Formulé de façon plus ou moins explicite dans divers écrits officiels, le skaz est enfin exposé à «l'état pur» dans le Préambule de la Constitution de 1977 qui proclame l'instauration de l'État-du-peuple-toutentier et renvoie dans un passé indéterminé la dictature du prolétariat, de sinistre mémoire. Ce skaz devient une fiche d'identité collective de l'URSS, il permet de situer dans le temps et dans l'espace l'Odyssée de cette héroïque armée de travailleurs en route vers l'avenir radieux. Systématiquement désinformés, les citoyens soviétiques sont-ils vraiment pressés de connaître de dures vérités? N’est-il pas préférable d'accepter une fiction qui, au moins, protège ceux qui la respectent des persécutions policières, celles dont sont victimes les dissidents, en quête de vérité ? Génératrice d'une stagnation qui met en péril l'avenir du pays, cette attitude apathique de la population ne pourra convenir au nouveau secrétaire général du PCUS, Gorbatchev, animé d'une "nouvelle pensée » et résolu à combattre la stagnation. Le discours soviétique va s'infléchir de façon imprévisible.

16 À partir du moment où notre Département de langues slaves, associé à son équivalent à Lyon 2, reçoit l'habilitation d'un $3^{e}$ cycle, notre séminaire du jeudi se transforme en cursus pour des étudiants engagés dans la rédaction d'une thèse de doctorat. J'en prends la direction, il va perdre le caractère d'improvisation qu'il avait, lorsque chacun de nous (nous étions peu nombreux) arrivait avec une nouvelle parution, Dire et ne pas dire d'Oswald Ducrot, Quand dire, c'est faire de J. L. Austin ou Langage et idéologie d'olivier Reboul. Les «anciens » se retrouvent désormais en réunion du comité de rédaction des Essais, pour examiner les articles proposés par des auteurs divers, Guy Bensimon, Jacqueline Fontaine, Charles Bourg, Françoise Adiba, Hélène Henry, Jean-Claude Lanne, Claude Robert, André Radiguet, Manuèle Jargot et bien d'autres, qui traitent du discours soviétique dans des contextes différents, traditionnel ou novateur : le cinéma soviétique, la Grande Guerre patriotique, totalitarisme et communisme, structure grammaticale et idéologie, le dit et le non-dit dans le discours sur Le Dit du Prince Igor, Gorbatchev et la " nouvelle pensée ", Zinoviev et le gorbatchévisme, les taches blanches et la réécriture de l'histoire, le décodage de La maison Pouchkine de A. Bitov, etc. Tendance ou dérive, l'orientation vers un patriotisme russe devient évidente. Dans le discours soviétique, il faut faire place à d'autres discours, d'où la nécessité de changer le titre des Essais à partir $\mathrm{du}^{\circ}{ }^{7}$, en 1987 : Essais sur le discours soviétique, russe et autres discours slaves. Novo Tomic et V.-C. Fisera attirent notamment l'attention sur la Yougoslavie, le destin historique et politique du serbo-croate, les convulsions ethno-linguistiques.

Les Essais s'enrichissent aussi de nouveautés, notamment d'illustrations: Les Russes de Gustave Doré, d'actualité, lors de la guerre de Crimée. L'œuvre picturale d'Ilia Glazounov, "L'apport des peuples de l'URSS à la culture et à la civilisation mondiales ", commentée par Alexis Berelowitch. Des caricatures de Mad sur l'actualité en URSS dans les années 1920 et 1930, publiées dans la Russie illustrée, l'hebdomadaire de l'émigration russe à Paris. Des photos de la revue, L'URSS en Construction de 1949, exaltant les succès de la science soviétique au service de l'homme. Les lettres de Russie d'une lectrice française. L'avant-garde soviétique ou plutôt l'Ivan-garde, d'après les photos de Valery Koutchenkov : le laborieux bricolage de clôtures de jardin, promues œuvres d'art.

L'analyse du discours soviétique devient un produit exportable. Je participe à des conférences en Allemagne, à Cologne, à Regensburg, à Heidelberg, d'où j'observe, avec les 
collègues allemands, l'évolution de la perestroïka et de son interprétation discursive. La lecture des Essais attire à Grenoble Ian Procop, professeur polonais de littérature comparée, détaché en Italie; grâce à lui, nous établissons des relations suivies avec l'université Jagellon à Cracovie. De même avec la Bulgarie, grâce à une doctorante, Margarita Vassileva. De même avec la Hongrie, la Tchécoslovaquie, à l'occasion de colloques de linguistique slave qui donnent l'occasion d'affronter le skaz soviétique officiel avec des arguments désormais bien rodés. Le CESC devient un pôle d'attraction pour de nombreux collègues étrangers désireux de coopérer avec nous. Nous préparons ensemble l'organisation à Grenoble d'un colloque qui réunirait des spécialistes du discours soviétique venant de l'Est et de l'Ouest, mais la chute du mur de Berlin précipite les événements et transforme un projet modeste en un Forum international. Il reçoit d'abondants subsides pour faire venir à Grenoble, sous le signe de la francophonie, des universitaires de tous les pays de «l'autre Europe » et des universitaires occidentaux. Du 31 janvier au 2 février 1990, se tient en France, à Grenoble, le premier grand Colloque universitaire Est-Ouest, sous une présidence ministérielle. Un attaché du ministre, un peu cynique, me glisse, admiratif : «Vous avez bien choisi le moment pour organiser votre colloque. » Je lui réponds : «Cela fait des années qu'on le prépare!»

Le discours soviétique a perdu ses repères, la "polyphonie démocratique » qui s'installe aboutit à une situation tragi-comique, où il devient impossible de reconnaître une droite ou une gauche; je traite avec ironie ce thème dans mon intervention «Perestroika as Revolution or Normalisation ", à Harrogate, au $4^{\mathrm{e}}$ World Congress for Soviet and East European Studies. La même année, en 1990, le coup de grâce au discours soviétique n'est pas porté par le CESC, mais par le Premier secrétaire de l'Ambassade, en visite traditionnelle à notre université. Informé au préalable sur nos " activités subversives ", il avait bien préparé son intervention. Inopinément, au cours de la réunion commune de nos étudiants français et soviétiques, il déclare : «Je voudrais apporter ma contribution à vos travaux sur notre discours officiel », déplie avec un air malicieux un papier et se met à citer et à commenter une liste d'expressions qui provoquent un rire sincère et libérateur dans toute l'assistance.

Quelle époque! Le discours soviétique est mort, la soviétologie est morte, le répertoire détaillé de la nomenklatura soviétique, un effort d'érudition colossal accompli par Michel Tatu, est devenu obsolète, mes outils d'analyse, le programme narratif de Greimas, son carré sémantique, son schéma de communication sont promis à l'oubli ou à d'autres usages, nos Essais changent encore de titre et deviennent Essais sur le discours de l'Europe éclatée, pendant que, regroupés dans des séminaires communs, mes étudiants en DEA et mes étudiants de Sciences Po, partent, pleins d'espoir, à la découverte de cette autre Europe.

\section{BIBLIOGRAPHIE}

Annuaire de l'URSS - Droit, Politique, Sociologie, Politique, Culture, Paris : Éditions du CNRS.

Austin John Langshow (1970), Quand dire, c'est faire, Paris : Éditions du Seuil. 
AlthUSSER Louis (1965), Pour Marx, Paris : Maspero.

BAКHTIN Mikhaïl (1963), ПРОБЛЕМЫ ПОЭТИКИ ДОСТОЕВСКОГО, Moscou : СОВЕТСКИЙ ПИСАТЕЛЬ.

BESANçon Alain (1977), Les origines intellectuelles du léninisme, Paris : Calmann-Lévy.

ВОПРОСЫ ФИЛОСОФИИ АНСССР, MOscou : ИЗД. ПРАВДА

BOURMEYSTER Alexandre (1974), Stankevic et l'idéalisme humanitaire des années 1830, Université

Lille 3.

DUCROT Oswald (1972), Dire et ne pas dire, Paris : Hermann.

Essais sur le discours soviétique (sémiologie, linguistique, analyse discursive), CESC, Université StendhalGrenoble 3.

GREIMAS Algirdas Julien (1966), Sémantique structurale, Paris : Larousse.

GREIMAS Algirdas Julien (1970), Du sens, Paris : Éditions du Seuil.

HELLER Mikhaïl (1980), « РУСсКИЙ ЯЗЫК И СОВЕТсКИЙ ЯЗЫК », РУССКАЯ МЫсЛЬ, Paris, 8 mai 1980.

JAKOBSON Roman (1963), Essais de linguistique générale, Paris : Éditions de Minuit.

КОММУНИСТ ТЕОРЕТИЧЕСКИЙ И ПОЛИТИЧЕСКИЙ ЖУРНАЛ ЦК КПСС, Moscou : ИЗД. ПРАВДА.

LÉVI-STRAuss Claude (1958), Anthropologie structurale, Paris : Plon.

ОЖЕГОВ С.И. (1960), СЛОВАРЬ РУССКОГО ЯЗЫКА, ИЗД. 4. Moscou : ГОСИЗДАТ ИНОСТР. И НАЦ.

СЛОВАРЕЙ.

PÊCHeUX Michel (1975), Les Vérités de La Palice, Paris : Maspero.

Propp Vladimir (1965), Morphologie du conte, Paris : Éditions du Seuil.

ReBoul Olivier (1980), Langage et idéologie, Paris : PUF.

SAUSSURE Ferdinand de (1955), Cours de linguistique générale, Paris : Payot.

SÉRIOT Patrick (1985), Analyse du discours politique soviétique, Institut d'études slaves.

Tном Françoise (1987), La langue de bois, Commentaire Julliard, Paris.

\section{ANNEXES}

\section{Le carré sémantique}




$\begin{array}{cccc}\text { Axe positif } & \begin{array}{c}\mathrm{A} \\ (\text { Liberté })\end{array} & & \begin{array}{c}\text { non A } \\ (\text { Ordre })\end{array} \\ \text { Axe négatif } & \frac{}{\text { non-A }} & \mathrm{X} & \overline{\mathrm{A}} \\ (\text { Désordre }) & & \text { (Despotisme) }\end{array}$

A et non A axe des contraires, relation de contrariété, présupposition réciproque susceptibles d'être présents de façon concomitante d'être vrais ou faux ensemble

$\mathrm{A}$ et $\overline{\mathrm{A}}$, non $\mathrm{A}$ et $\overline{\text { non } \mathrm{A}}$ contradiction par négation, impossibilité d'être présents ensemble.

Réduction unilatérale du carré sémantique à une alternative :

Discours libéral : soit liberté, soit despotisme (ordre identifié à despotisme; désordre, non-dit). Discours autoritaire : soit ordre, soit désordre (liberté identifiée à désordre, despotisme, non-dit).

$\begin{array}{cccc}\text { Liberté } & \begin{array}{c}\text { pouvoir accepter } \\ \text { (adhérer) }\end{array} & \begin{array}{c}\text { pouvoir refuser } \\ \text { (choisir) }\end{array} \\ \text { Obligation } & \begin{array}{c}\text { devoir accepter } \\ \text { (obéir) }\end{array} & & \begin{array}{c}\text { devoir refuser } \\ \text { (renoncer) }\end{array} \\ \text { parler vrai } & & \text { mentir } \\ \text { Démentir } & \mathrm{X} & \text { feindre }\end{array}$

Je laisse au lecteur le soin d'appliquer le carré sémantique au cas de Raskolnikov. Il devrait le conduire au suicide. Dostoïevski lui concède une sortie et sacrifie à sa place son double Svidrigaïlov.

\section{RÉSUMÉS}

Le compte rendu des articles de Voprosy filosofii et Kommunist, pour l'Annuaire de l'URSS (CNRS, Strasbourg), ensuite l'exploitation pédagogique de la presse soviétique, la Pravda, les Izvestia, à l'université de Grenoble, me révèlent la singularité du discours soviétique. Il mérite une approche universitaire, critique, certes, mais dénuée d'a priori politique. La Morphologie du conte de Vladimir Propp, les Problèmes de la poétique de Dostoïevski de Mikhaïl Bakhtin, la sémiotique et le programme narratif de Greimas, le schéma de communication de Jakobson servent de fondement à la création en 1978, au Centre d'études slaves contemporaines, d'un séminaire de $3^{\mathrm{e}}$ cycle sur le «Discours soviétique ». Se référant à Michel Pêcheux qui fonde sa réflexion sur la notion de coupure épistémologique, héritée de Bachelard, Patrick Sériot écarte les approches "subjectives » de la phraséologie soviétique, celles de Michel Heller, d'Alain Besançon ou de Françoise Thom qui prétend traiter grammaticalement la «langue de bois »! En 1981, sort le premier numéro des Essais sur le discours soviétique. En 1983, à Grenoble, grâce à l'informatique, Patrick Sériot révèle dans sa thèse, une manipulation anonyme, destinée à effacer toute référence à des prédications antérieures; il aboutit, sans définition a priori, à différencier langue et discours. Au $3^{\mathrm{e}}$ Congrès mondial d'études soviétiques et est-européennes à Washington en 1985, j'expose ma théorie du skaz, une fiche d'identité collective de l'URSS, situant dans le temps et dans l'espace l'odyssée d'une héroïque armée de travailleurs, en route vers l'avenir radieux. Nous observons l'usage de cette fiction par les étudiants soviétiques en stage chez nous à Grenoble. Le CESC acquiert un statut international avec l'habilitation d'un DEA russe et langues slaves Grenoble-Lyon. Il poursuit la publication annuelle des Essais sur le discours soviétique et coopère avec des collègues de l'Europe de l'Est. Début 1990, à Grenoble, se tient sous son égide le premier grand Colloque 
universitaire Est-Ouest. Mais avec l'Union soviétique meurent simultanément soviétologie et discours soviétique, nos Essais deviennent Essais sur le discours de l'Europe éclatée, pendant que nos étudiants partent à la découverte de cette autre Europe.

The review of articles from Voprosy filosofii and Kommunist for the Annuaire de l'URSS (CNRS, Strasbourg), followed by the pedagogical exploitation of the Soviet Press (Pravda and Izvestia) brought into light the singular nature of the Soviet discourse. It deserved an academic approach that could be critical but free from politically-inspired preconceptions. The Folk Tale Morphology by Vladimir Propp, The Problems of Dostoievski's Poetics by Mikhail Bakhtin, Greimas's semiotics and narrative programme, Jakobson's communication pattern served as the basis for the creation of a Graduate Seminar on Soviet Discourse in the Centre d'Études Slaves Contemporaines in 1978. Referring to Michel Pêcheux, whose own reflection is founded on the concept of epistemological fracture inherited from Bachelard, Patrick Sériot turned away from the "subjective" approach of Soviet phraseology, like those of Michel Heller, Alain Besançon or Françoise Thom who claims she can take a grammatical approach to newspeak! In 1983, in Grenoble, thanks to computer science, Patrick Sériot unveiled in his dissertation an anonymous manipulation aimed at erasing any reference to previous pronouncements and, without any a priori definition, ended up differentiating language and discourse. At the 3rd World Conference on Soviet and East European Studies in Washington in 1985, I gave a paper on my theory of skaz, a collective identity card of the the USSR that situates in time and space the odyssey of a heroic army of workers on their way to a shining horizon We observed the usage of this fiction by the Soviet students undergoing a training period at our university in Grenoble. The CESC acquired an international status when the joint Grenoble-Lyon DEA (pre-doctoral) degree of Slavic studies was granted ministerial accreditation. It then continued the annual publication of Essays on the Soviet Discourse and cooperates with Eastern European colleagues. In early 1990, the first major East-West academic conference was held under its patronage in Grenoble. But with the simultaneous demise of the Soviet Union, sovietology, and the Soviet discourse, our Essays became the Essays on the discourse of a fragmented Europe, while our students set out to discover this other Europe.

\section{INDEX}

Keywords : language, discourse, inter-discourse, semiotics, skaz

Mots-clés : langue, discours, inter-discours, sémiologie, skaz

\section{AUTEUR}

\section{ALEXANDRE BOURMEYSTER}

Professeur émérite, Université Grenoble Alpes, ILCEA 4 (CESC) 